\section{Relationship between satisfaction with adolescent extracurricular activities and social withdrawal: The mediating effect of self-esteem and sense of community}

Cho Yeon Soo $\cdot$ Han Sae-Young ${ }^{1}$

Ewha Womans University, Dept. of Child Development, Student; ${ }^{1}$ Ewha Womans University, Dept. of Child Development, Professor

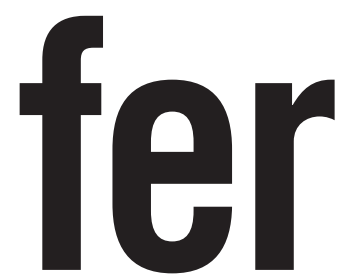

\section{Family and}

\section{Environment}

\section{Research}

청소년 체험활동 만족도가 사회적 위축에 미치는 영향: 자아존중감과 공동체의식의 매개효과

조연수 · 한세영 ${ }^{1}$

이화여자대학교 아동학과 학생, ${ }^{1}$ 이화여자대학교 아동학과 교수

\section{Abstract}

This study examines the effect of satisfaction with adolescent extracurricular activities on social withdrawal with a focus on the mediating effect of self-esteem and sense of community. We used data from the 3rd wave (2012) of the Korean Children and Youth Panel Survey (KCYPS) collected by the National Youth Policy Institute for path analysis. Participants were 1,139 first year middle school students who participated in adolescent extracurricular activities. First, the degree of satisfaction with the adolescent extracurricular activities had an indirect effect on social withdrawal via self-esteem. Second, satisfaction with the adolescent extracurricular activities had an indirect effect on social withdrawal via a sense of community. Lastly, satisfaction with adolescent extracurricular activities had an indirect effect on social withdrawal via self-esteem and a sense of community. The research findings indicate that the level of social withdrawal is less when adolescents are satisfied with adolescent extracurricular activities and have a high level of self-esteem and sense of community. The results demonstrate that a satisfactory level of adolescent extracurricular activity would help adolescents decrease their social withdrawal by increasing the level of self-esteem and sense of community.

Keywords

adolescence, satisfaction with extracurricular activities, social withdrawal, self-esteem, sense of community

\section{Introduction}

It was presented at the poster session at the 19th Biennial International Congress Asian Regional Association for Home Economics (ARAHE) in 2017.

\section{Corresponding Author:}

Han, Sae-Young

Ewha Womans University

Child Development

Professor

52, Ewhayeodae-gil, Seodaemun-gu,

Seoul, Republic of Korea

Tel: $+82-2-3277-4380$

Fax: +82-2-3277-2783

E-mail: evenhow@ewha.ac.kr 
social withdrawal, caused by over-controlled behavior (Mash \& Barkely, 2003). The importance of internalizing problems has not been investigated compared to the amount of study on externalizing problems (Seo, 2008). However, there is a need for research on internalizing problems in Korea, because $11 \%$ of adolescents suffer from internalizing problems such as social withdrawal and depression (externalizing symptoms, 4\%)(Seoul National University Hospital, 2018).

People who have been isolated from society often show a tendency towards social withdrawal that involves avoiding direct contact with other people. Social withdrawal is a typical internalizing problem, along with anxiety and depression; however, there are few studies that focus on social withdrawal compared with those that focus on depression and anxiety (Jwa \& Oh, 2011). According to a series of studies on social withdrawal (Rubin \& Burgess, 2001; Rubin, Chen, \& Hymel, 1993; Shin, 2007), it is reported that social withdrawal is strongly correlated with psychological problems such as depression, anxiety, and low self-esteem; in addition, chronic social withdrawal is reportedly correlated with critical social problems, such as social isolation, criminal behavior, and suicide (Coplan et al., 2013; Oh et al., 2008; Rubin, Coplan, \& Bowker, 2009; Schwartz, Snidman, \& Kagan, 1999), as well as psychological disorders such as avoidant personality disorder, social phobia, autism spectrum disorder, and schizophrenia (Coplan et al., 2013). Changes in the social structure, caused by cell phones, the Internet, and the expansion of nuclear families, have also led to an alone culture in Korea with interruptions of direct communication among people. Accordingly, increased levels of social withdrawal have arisen as well and become a severe social problem; consequently, the importance of investigating social withdrawal in adolescents has come to the fore based on reports that social withdrawal among adolescents is increasing. Korean adolescents lack opportunities to engage in communication with families and peer groups due to excessive social pressure with regard to academic achievements, which may lead them to suffer social withdrawal (Yang, Kim, \& Oh, 2006). It is therefore necessary to explore variables that can predict social withdrawal.

According to ecological systems theory of Bronfenbrenner (1989), the qualities of interaction between children and their environment may influence development. The social development of adolescents can be influenced by interaction with a multiple level of environments. Research on youth activities is increasing in regards to promoting experience with social interaction. According to Brown (2007), it is crucial for adolescents to engage in extracurricular activities so as to enter a healthy adulthood by gaining stability in terms of physical, psychological, and social development. Adolescents can experience the process of socialization through various activities in diverse groups and master the virtues that they must learn as members of society. They may also learn prosocial behaviors that are essential to grow into adulthood through various social relationships and communal living experiences beyond family relations (Hwang, Huh, \& An, 2013). Moreover, precedent studies indicate that various types of extracurricular activities by adolescents can reduce internalizing problems, including social withdrawal (Bohnert, Kane \& Gaber, 2008; Lee, Jung, \& Lee, 2014; Lee, Lee, \& Han, 2015).

It is also necessary to investigate the role of the social self as well as the individual self as mediating variables between social activities and social withdrawal symptoms in social relations. The individual develops the ego by interacting with other members in society to which it belongs to because the ego can be encouraged or discouraged by activities and feelings that arise from activities in one's social group. These experiences with activities may lead to changes in a sense of belonging to the group and identity as a member that further leads to greater or withering interpersonal confidence. Several studies have shown that participating in extracurricular activities leads to greater self-esteem through a sense of accomplishment stemming from the participation in an activity (Choi, 2002; Choi, 2014; Kim, 2007; Ryu \& Kang, 2012). It is also reported that adolescents who had higher satisfaction 
with extracurricular activities showed higher self-esteem while there was no significant difference in self-esteem between adolescents who spent more time and those who spent less time on extracurricular activities (Hwang, Huh $\&$ An, 2013). The finding implies that the qualitative aspect of adolescent extracurricular activities may have a more important role in raising self-esteem, a psychological evaluation, than the quantitative aspect of them, such as the frequency of participation. Rosenberg (1979) defines self-esteem as a positive or negative attitude about the self. While high self-esteem plays a positive role in adolescents' personal development and social adjustment (Hwang, Huh \& An., 2013), low self-esteem is related to negative selfunderstanding and self-consciousness with a lack of social ability that often leads to social withdrawal (Altmann \& Gotlib, 1988). Earlier studies have consistently reported that self-esteem is closely related to social withdrawal (Park \& Hong, 2014) and that low self-esteem exacerbates social withdrawal (Park, 2014).

The psychological processes associated with social withdrawal include self-esteem, an evaluative emotion about oneself as well as a sense of community that refers to feelings about the relationship between oneself and society. A sense of community is a feeling described by belonging where members matter to one another and to the group, and with a shared faith that members' needs will be met through their commitment to be together (McMillan \& Chavis, 1986). In addition, adolescent extracurricular activities allow adolescents to cultivate value in their community and develop a sense of community. Precedent studies have demonstrated that the experience of adolescent extracurricular activities affects one's sense of community positively (Kim, 2014; Moon \& Moon, 2010; Park, 2009; Yoon, 2011), and adolescents who have a higher satisfaction with extracurricular activities show a higher sense of community (Hwang, Huh, \& An, 2013). Developing a sense of community can be a means to solve behavioral problems such as social withdrawal because a sense of community is based on an understanding of and sympathy towards others - beyond a single individual (Woo \& Nho, 2014).
It was also reported that a high sense of community in adolescents ameliorates negative emotions such as social loneliness (Chipuer, 2001), and the development of a sense of community in this age cohort is associated with the development of sociality (Cho, 2007). This indicates that a sense of community is a psychological reaction to shared responsibilities. In addition, the advantages and disadvantages of the community contribute to the cultivation of sociality and ameliorate symptoms such as social withdrawal by establishing mutual dependence, democracy, and openness based on a sound conscience, solidarity, and social responsibility (Park, 2009). Thus, this study tested how self-esteem and a sense of community, among various contextual factors which affect social withdrawal, mediate the relationship between adolescent extracurricular activities and social withdrawal.

Self-esteem is a mediating factor between extracurricular activities by adolescents and social withdrawal that is also related to a sense of community that is seen as another mediating factor. Studies on adolescent self-esteem and sense of community show that the two factors are significantly correlated (An \& Park, 2017; Du, Bernardo, \& Yeung, 2015; Kwon, 2014). Self-esteem was reported to influence a sense of community in most studies dealing with the relation between self-esteem and a sense of community (Hong, 2015; Jung, Kim, \& Kim, 2016; Kim \& Ahn, 2012; Yang \& Kim, 2015). A higher self-esteem in adolescents results in a higher sense of community. This relationship implies that the development of self-esteem, i.e., a positive perception of oneself, contributes to a sense of community that has positive perceptions of relationships with others or a group and a commitment based attitude to a community.

Few studies show the effect of satisfaction with adolescent extracurricular activities on social withdrawal directly. A precedent study on factors of social withdrawal in adolescence based on contextual perspective (Lee, Min \& Kim, 2014) showed that the strongest factor contributing to social withdrawal among contextual variables was personal variables such as depression and anxiety, and that the next important factors were family variables, such as level 
of parent educational participation, and peer relations. School variables and community variables were not significant variables in predicting social withdrawal. Therefore it can be assumed that there is no direct effect of satisfaction with extracurricular activities towards social withdrawal. Studies on the effects of adolescent extracurricular activities on adolescents' general levels of emotion and sociality indicate that possible effects can be identified as having two parts. The first pertains to the quantitative aspect of adolescent extracurricular activities that include participation, the number of activities, and time spent on each activity; the second refers to the qualitative aspects of adolescent extracurricular activities and satisfaction with activities. Research on adolescent extracurricular activities has focused on quantitative aspects with inconsistent outcomes. Several studies have reported that participation by adolescents in extracurricular activities affects sociality positively (Conrad \& Hedin, 1989; Do \& Sung, 2013; Ji, 2006; Kim, 1999; Kim et al., 2014); however, research has also showed no relationship between adolescent extracurricular activities and the development of sociality (Ryu, 2013). Studies also indicate that satisfaction with adolescent extracurricular activities may affect the social emotional development of adolescents as well as suggest a need for additional investigation that focuses on qualitative aspects of adolescent extracurricular activities. Research on the qualitative aspects of adolescent extracurricular activities is relatively scarce; however, some studies have found significant effects on the development of sociality, peer relations, personal relations and school adaptation (Choi, 2018; Park \& Lee, 2015; Ryu, 2013; Ryu \& Kang, 2012). It was also reported that adolescents who had a higher satisfaction with extracurricular activities showed a lower level of alienation from friends, while the amount of the time they spent on extracurricular activities had no significant effect (Hwang, Huh, \& An, 2013). Satisfaction with extracurricular activities is reported to influence adolescents' social and interpersonal development.

We can assume mediating paths among satisfaction with adolescent extracurricular activities, self-esteem, a sense of community, and social withdrawal. Paths among the factors exist in part; however, most research has focused on the quantitative aspects of adolescent extracurricular activities with few studies investigating the role of self-esteem, sense of community between adolescent extracurricular activities and social withdrawal. This study therefore examines the path by which satisfaction with adolescent extracurricular activities affects social withdrawal via self-esteem and a sense of community indirectly.

\section{Methods}

\section{Participants}

The data used in this study was the third wave data (2012) of '2010 Korean Children and Youth Panel Survey (KCYPS)' conducted by the National Youth Policy Institute (NYPI). The survey participants in 2010 were 2,351 first year middle school students recruited using a stratified multi-stage cluster sampling from 12 regions (Seoul Metropolitan City and 11 metropolitan cities and provinces) in South Korea. The survey investigated adolescents' experience with extracurricular activities that included 9 sections (health activities, scientific information activities, interchange activities, adventure and pioneering activities, culture and art activities, volunteer activities, work experience activities, environmental conservation activities, and self-improvement activities). However, the data used in this study is a sub-sample of the original data. Factor analysis was executed on nine sections of extracurricular activities, and only four sections of extracurricular activities, which were considered as social activities, are included in this study. Participants included in the study included 1,139 middle school adolescents who participated in at least one adolescent extracurricular activity such as interchange activities, adventure and pioneering activities, culture and art activities, and volunteer activities. Also, 1,212 adolescents who have never participated in the four sections of extracurricular activities were excluded from 
Table 1. Examples of Adolescent Extracurricular Activities

\begin{tabular}{|c|c|}
\hline Section & Examples \\
\hline 1. Interchange activities & $\begin{array}{l}\text { Adolescent international interchange activity, South and North Korean adolescent interchange activity, understanding } \\
\text { multi-culture activity. }\end{array}$ \\
\hline 2. Adventure and pioneering activities & Exploring/climbing activity, outdoor activity, marine activity, boot camp. \\
\hline 3. Culture and art activities & Local culture, world culture, public culture, historical play activity, traditional culture activity. \\
\hline 4. Volunteer activities & Volunteer assistance, teaching, campaign, relief activity. \\
\hline
\end{tabular}

Note. Table adapted from the ministry of gender equality and family of Republic of Korea (2012).

the analysis of this study. The portion of male (48.5\%) and female (51.5\%) participants were similar. As for parents' educational backgrounds, more than half of fathers (53.4\%) had college or higher educational background, and approximately half of mothers (49.7\%) had high school education or less. The annual average family income was about 48 million KRW.

\section{Measures}

\section{1) Satisfaction with adolescent extracurricular activities}

Adolescents responded to questions developed by the Korean Youth Panel Survey (KYPS) and revised by the National Youth Policy Institute (NYPI) on their satisfaction with extracurricular activities. Respondents rated satisfaction with adolescent extracurricular activities that included interchange activities, adventure and pioneering activities, culture and art activities, and volunteer activities (Table 1), using four-point rating scales with points ranging from 1 (never satisfied) to 4 (very satisfied). Higher scores reflect higher levels of extracurricular activity satisfaction. The mean satisfaction score from the four types of activity was used in the analysis; in addition, the internal reliability of this scale was good (Cronbach's alpha $=.85$ ).

\section{2) Self-esteem}

Self-esteem was defined as a positive or negative attitude about the self according to the definition by Rosenberg (1979). Adolescents reported on self-esteem using Rosenberg's (1965) Self-Esteem Scale, which was translated by the Behavioral Science Research Center of Korea University. They were asked to respond to five positive items and five negative items on a four-point scale ranging from strongly agree to strongly disagree (e.g., "On the whole, I am satisfied with myself.", "I feel that I have a number of good qualities", and "All in all, I am inclined to feel that I am a failure.”). Negative items were reversescored with a higher score indicating higher self-esteem. Nine items were used in the analysis after the statement "I wish I could have more respect for myself." was removed due to ambiguity that lowered the overall reliability of the internal reliability of the scale. The internal reliability (Cronbach's alpha) of this scale was .87.

\section{3) Sense of community}

This study defined a sense of community as devoted attitudes and behavior to one's community that surpasses egoism. A sense of community was measured using the Democratic Civic Consciousness Questionnaire developed by Kwon (2004), which was revised by the National Youth Policy Institute (NYPI). This questionnaire included four items (e.g., "I can help my friend in need actively." and "I can volunteer at welfare institutions on national holidays instead of taking a rest."). These items used a four-point Likert scale ranging from 1 (strongly disagree) to 4 (strongly agree), and higher scores were indicative of a higher sense of community. The internal reliability (Cronbach's alpha) of the scale was .80 .

\section{4) Social withdrawal}

Social withdrawal is defined as a tendency to shrink from social situations. Adolescents reported on social 
Family and Environment Research

Table 2. Correlations among Variables $(N=1,139)$

\begin{tabular}{|c|c|c|c|c|}
\hline Variables & 1 & 2 & 3 & 4 \\
\hline 1 Satisfaction of adolescent extracurricular activity & - & & & \\
\hline 2 Self-esteem & $.12^{*}$ & - & & \\
\hline 3 Sense of community & $.21^{*}$ & .24 & - & \\
\hline 4 Social withdrawal & -.04 & $-.41^{\prime \prime}$ & $-.18^{\prime \prime}$ & - \\
\hline M & 3.23 & 2.86 & 2.89 & 2.23 \\
\hline SD & .56 & .46 & .56 & .73 \\
\hline
\end{tabular}

$" p<.01$

withdrawal using a five-item measure. The measurement is a revised version of 'Development of behavior problem scale for children and adolescence' (Kim \& Kim, 1998) by the National Youth Policy Institute (NYPI) (e.g., "I am shy.", "It's difficult to express my opinion clearly.", and "I don't like to stand out."). A four-point Likert scale ranging from 1 (strongly disagree) to 4 (strongly agree) was used. Higher scores reflect a perception of higher social withdrawal. The internal reliability (Cronbach's alpha) of the scale was 89.

\section{5) Control variable}

As control variables, household income, maternal educational level, and paternal educational level were included, because precedent studies (Cho, Lee, \& Ko, 2014; Seo, 2007) reported that the parents'socioeconomic status had influence on the leisure activities of adolescents.

\section{Procedure}

Data analyses were conducted by using SPSS 21.0 (SPSS Inc., Chicago, IL, USA) and AMOS 21.0 (SPSS Inc, Chicago, IL, USA) programs. First, descriptive statistics and correlations were used to analyze the data with SPSS 21.0. Second, a path analysis model examined how a sense of self-esteem and a sense of community mediated the relationship between satisfaction with adolescent extracurricular activities and social withdrawal. The model was tested using Amos version 21.0 (Arbuckle, 2012). Lastly, a bootstrapping method was used to confirm mediation effects.

\section{Results}

\section{Correlations among all variables}

Table 2 presents the correlations between the variables. All variables except for social withdrawal were significantly correlated with each other. First, satisfaction with adolescent extracurricular activities was positively correlated with a sense of self-esteem $(r=.12, p<.01)$, a sense of community $(r=.21, p<.01)$, but not correlated with social withdrawal. Also, a sense of self-esteem was positively related to a sense of community $(r=.24, p<.01)$, and negatively correlated with social withdrawal ( $r=-$ $.41, p<.01)$. Lastly, a sense of community was negatively associated with social withdrawal $(r=-.18, p<.01)$.

\section{Mediation Processes}

The goodness of model fit was tested to examine how well the model fits the data. Fit indices such as $\chi 2$, GFI, NFI, TLI, CFI, and RMSEA examined the goodness of model fit. According to cutoff criteria for fit indices (Hong, 2000), the model fit was considered acceptable with NFI $>$.90, TLI $>$.90, CFI .90 and RMSEA<.05. The goodness-of-fit showed an adequate fit $\chi 2(1, N=1,139)=2.528, \mathrm{NFI}=.994$, TLI=.977, CFI=.996, RMSEA $(90 \%$ CI $)=.034(.000, .088)$ except for the figure of $\chi^{2}$. It is a good model fit when $\chi^{2}$ is not significant statistically, and the figure of $\chi^{2} / d f$ is between 2 and 5 (Marsh \& Hocevar, 1988), the figure of $\chi^{2}$ is easily influenced by sample size; therefore, it is necessary to consider other goodness-of-fit indices collectively (Kim, 2010). Table 3 displays the goodness-of-fit indices.

The result of path analysis is presented in Table 4 
Table 3. Model Fit $(N=1,139)$

\begin{tabular}{ccccccc}
\hline$\chi^{2}(d f)$ & $\chi^{2} / d f$ & GFI & NFI & TLI & CFI & RMSEA (LO90 HI90) \\
\hline $33.790^{* * * *}(12)$ & 2.816 & .992 & .976 & .972 & .984 & $.040(.024 \sim 056)$ \\
\hline
\end{tabular}

$p<.001$.

and Figure 1. First, regarding the indirect path that the satisfaction with adolescent extracurricular activities affected social withdrawal via self-esteem, satisfaction with adolescent extracurricular activities was positively associated with a sense of self-esteem $(\beta=.12, p<.001)$, and a sense of self-esteem was negatively associated with social withdrawal $(\beta=-.39, p<.001)$. Second, regarding the indirect path by which satisfaction with adolescent extracurricular activities affected social withdrawal via a sense of community, satisfaction with adolescent extracurricular activities was positively associated with a sense of community $(\beta=.186, p<.001)$, and a sense of community was negatively associated with social withdrawal $(\beta=-.094, p<.001)$. Third, regarding the indirect path by which satisfaction with adolescent extracurricular activities affected social withdrawal via a sense of self-esteem and a sense of community, satisfaction with adolescent extracurricular activities was positively associated with a sense of self-esteem $(\beta=.118$, $p<.001$ ), and a sense of self-esteem was positively associated with a sense of community $(\beta=.214, p<.001)$, which had a negative impact on social withdrawal ( $\beta$ $=-.094, p<.001)$. Adolescents who had higher levels of satisfaction with adolescent extracurricular activities had higher levels of self-esteem and sense of community,

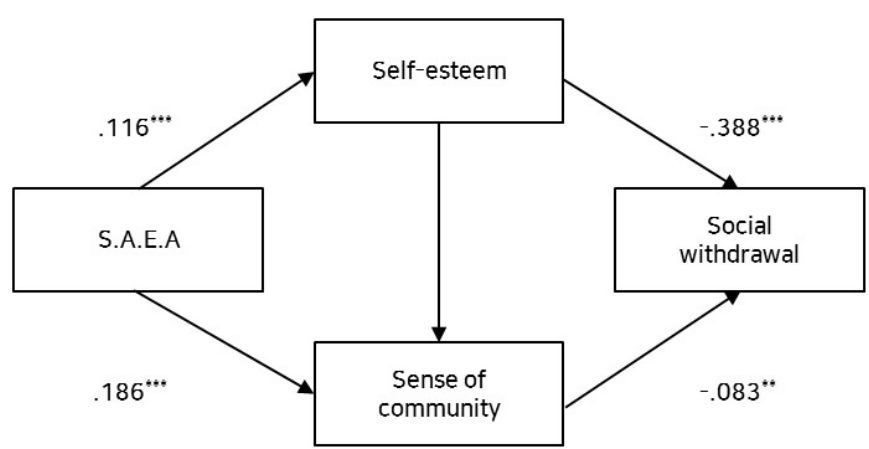

Figure 1. Relation between the satisfaction of adolescent extracurricular activity, self-esteem, sense of community, and social withdrawal.

Note: Household income, maternal educational background, and paternal educational background are included as control variables. S.A.E.A = Satisfaction with adolescent extracurricular activities.

"*m $p<.001 ., " p<.01$.

which led to less social withdrawal. Lastly, Table 5 verifies the statistical significance of the mediating effects.

\section{Discussion}

This study investigated the indirect effect of satisfaction with adolescent extracurricular activities on social withdrawal through self-esteem and a sense of community

Table 4. Path Model Results

\begin{tabular}{lcccc}
\hline & $b$ & $\beta$ & S.E & C.R \\
\hline S.A.E.A $\rightarrow$ self-esteem & $.095^{* * *}$ & .116 & .024 & 3.938 \\
S.A.E.A $\rightarrow$ sense of community & $.184^{* * *}$ & .186 & .184 & 6.533 \\
Self-esteem $\rightarrow$ sense of community & $.264^{* * *}$ & .220 & .034 & 7.727 \\
Self-esteem $\rightarrow$ social withdrawal & $-.623^{* * *}$ & -.390 & .044 & -14.031 \\
Sense of community $\rightarrow$ social withdrawal & $-.117^{* *}$ & -.088 & .037 \\
\hline
\end{tabular}

Note: Household income, maternal educational background, and paternal educational background are included as control variables. S.A.E.A = Satisfaction with adolescent extracurricular activities.

$p<.001 . " p<.01$. 
Family and Environment Research

Table 5. Direct, Indirect, and Total Effects of Relationship between the Variables

\begin{tabular}{|c|c|c|c|c|}
\hline & Direct effect & Indirect effect & Total effect & SMC \\
\hline S.A.E.A $\rightarrow$ social withdrawal & .000 & $-.063^{*+*}$ & $-.063^{* \prime \prime}$ & .173 \\
\hline Self-esteem $\rightarrow$ social withdrawal & $-.388^{* * \prime}$ & $-.018^{* *}$ & $-.407^{-*}$ & \\
\hline Sense of community $\rightarrow$ social withdrawal & $-.083^{*}$ & - & $-.083^{\prime \prime}$ & \\
\hline S.A.E.A $\rightarrow$ sense of community & $.186^{\prime \prime \prime}$ & $.025^{\prime \prime}$ & $.211^{\prime * *}$ & .092 \\
\hline Self-esteem $\rightarrow$ sense of community & $.220^{*+*}$ & - & $.220^{\prime \prime \prime}$ & \\
\hline S.A.E.A $\rightarrow$ self-esteem & $.116^{\prime \prime \prime}$ & - & .116 & .013 \\
\hline
\end{tabular}

Note: S.A.E.A = Satisfaction with adolescent extracurricular activities. SMC: Squared multiple correlation .

$p<.001 . " p<.01$.

in adolescent respondents. The current project used data from the third wave of in the 2010 Korean Children and Youth Panel Survey (KCYPS) that consisted of 1,139 adolescent participants who were in their first year of middle school in 2010.

First, satisfaction with adolescent extracurricular activities had an indirect effect on social withdrawal via self-esteem. Adolescents who displayed greater levels of satisfaction with extracurricular activities also had higher levels of self-esteem, which led to less social withdrawal. In addition, adolescents who perceived higher levels of satisfaction with adolescent extracurricular activities had greater self-esteem, which matched the findings of earlier research that reported adolescents who had a higher satisfaction with extracurricular activities had higher self-esteem (Hwang, Huh \& An, 2013). The finding that adolescent self-esteem affected the degree of social withdrawal is also consistent with a previous study that reported that low self-esteem in children exacerbated social withdrawal (Park, 2014). This finding is consistent with previous studies showing that internalizing problems such as social withdrawal, depression, and anxiety is strongly associated with low self-esteem (Kuhlberg, Pena, \& Zayas, 2010; McDonald et al., 2005; Smokowski, Rose, \& Bacallao, 2009).

The more adolescents perceived satisfaction with extracurricular activities, the greater their perceived self-esteem, which is linked to lower social withdrawal. Adolescents are likely to achieve greater self-esteem based on positive feelings of self-worth and competence if they feel a strong satisfaction towards extracurricular activities. Adolescents who have higher self-esteem also may reduce the tendency that they become sensitive to the negative reactions of others excessively (Gunnar \& Quevedo, 2007), possibly lowering the potential to suffer social withdrawal. The findings provide a potential mechanism for developing a social withdrawal intervention mechanism.

Second, satisfaction with adolescent extracurricular activities as perceived by adolescents affected social withdrawal via a sense of community. The more adolescents perceived satisfaction with adolescent extracurricular activities, the greater their perceived a sense of community, leading to less social withdrawal. Adolescents who had greater satisfaction with adolescent extracurricular activities perceived a greater sense of community that follows earlier works which indicated adolescents who had a higher satisfaction with extracurricular activities showed a higher sense of community (Hwang, Huh, \& An, 2013). In addition, the result is line with the study that groups of adolescents taking part in more social participation and volunteering more frequently had a higher sense of community (Kim, 2014). This result suggests that the more adolescents are satisfied with adolescent extracurricular activities, the more they can have positive values and attitudes to their community by experiencing acceptance by others in the community. The result that a sense of community by adolescents significantly affected the degree of social withdrawal is similar to research which indicated 
an adolescents' sense of fellowship with their school and community was associated with social loneliness (Chipuer, 2001). This demonstrates that improving adolescents' sense of community will allow them to internalize the value of coexistence that leads to positive attitudes towards interpersonal relations and lowers their levels of social withdrawal. The results imply that developing a sense of community is a significant process to ameliorate adolescents' social withdrawal.

Satisfaction with adolescent extracurricular activities affected social withdrawal via a sense of community. The more adolescents perceived satisfaction with an adolescent extracurricular activity the more they perceived a sense of community, which encouraged less social withdrawal. This result follows previous studies which reported that groups that had more participated in adolescent activities (higher participation rates and longer time spent) in the first and second years showed higher social relationship development in the third year (Hwang \& An, 2013) and that the peer relationships of adolescents who participated in an adolescent swimming class had a positive effect on a sense of community and improved their social maturity (Kim \& Yoo, 2009). The results indicate that the pleasurable perception and experience of an adolescent extracurricular activity enhanced the level of group solidarity, which contributed to promoting a sense of community and assertive social participation, lessening social withdrawal. This suggests a need in adolescents to consider satisfaction with extracurricular activities but also a sense of community when examining social withdrawal. In addition, the experience of adolescent extracurricular activities to promote a sense of community is meaningful to prevent social withdrawal or treat it when it does occur.

Third, among the paths in our research model, satisfaction with adolescent extracurricular activities was associated with self-esteem that first affected a sense of community and then social withdrawal. In this process, adolescents' self-esteem, one mediator variable, affected a sense of community, another mediator variable. This result is in line with studies that showed adolescents who perceived self-esteem positively had higher perceptions of a sense of community (Kwon, 2014; Moon \& Moon, 2009; Park, 2009). This is also provided evidence that when adolescents considered themselves competent and valuable, they were able to create a close bond with members of a community that increased their sense of community because they feel a sense of belonging and a willingness to devote themselves to a community.

Self-esteem had a greater influence on social withdrawal than a sense of community in the relationship among satisfaction with adolescent extracurricular activities, selfesteem, a sense of community, and social withdrawal. This result indicates that it is essential for adolescents to improve self-esteem when intervening in social withdrawal and that the inner self is a more powerful factor in reducing social withdrawal. This result can contribute to areas of counseling and educational settings.

There are several limitations of this study as well as valid proposals for future work. First, the main variables selected for this study used a simplified questionnaire conducted by KCYPS; therefore, further studies can scrutinize the relationships among variables by in-depth measurements. Second, this study has limitations regarding the crosssectional design used to examine only students in the first year of middle school. Accordingly, further work in the form of longitudinal studies can examine relationships between earlier extracurricular activities and subsequent effect by such activities on social withdrawal. Lastly, this study has the potential problem of shared variance due to the use of a self-report survey by adolescents. Thus, future studies could use multiple assessments with more objective measurements.

Despite the limitations, these findings have several important implications. First, this study can be generalized due to its use of panel data from a representative sample gathered using a stratified sampling method of the 2010 Korean Children and Youth Panel Survey (KCYPS). Second, we investigated adolescent extracurricular activities as antecedents that influence social withdrawal due to the lack of research on emotions. It was also valuable to find a 
mediating role of self-esteem, which is an inner-personal factor, as well as a sense of community, an evaluation of relationships with group and group members, shown between adolescent extracurricular activity and social withdrawal. Adolescents who have high self-esteem can overcome emotional problems through positive evaluations of extracurricular activities rather than experiential factors by perceiving a sense of community through a positive sense of fellowship and belonging. In addition, it is significant for adolescents to feel emotional satisfaction through highquality extracurricular activities and to cultivate sociality based on relationships with others in order to develop positive self-esteem and a sense of community. Third, precedent studies have suggested the importance of the quantitative aspects of adolescent extracurricular activities, such as participation and length of participation; however, this study found a significant influence in the qualitative aspects of adolescent extracurricular activities on adolescent emotional problems. Lastly, this study used the overall average of the satisfaction score of four sections of adolescent extracurricular activities determined as social activities to draw a broader conclusion not limited to one area. The findings from this study provide basic information to develop policies about adolescent extracurricular activities that assist the efforts of adolescents to develop socioemotionally as well as help develop intervention programs to protect adolescents from the risks of social withdrawal.

\section{Declaration of Conflicting Interests}

The authors declare no conflict of interest with respect to the authorship or publication of this paper.

\section{References}

Adamski, D. (2018). The influence of new technologies on the social withdrawal (Hikikomori syndrome) among developed communities, including Poland. Social Communication. Online Journal, 1(17), 5863. https://doi.org/10.2478/sc-2018-0007

Altmann, E. O., \&t Gotlib, I. H. (1988). The social behavior of depressed children: An observational study. Journal of Abnormal Child Psychology, 16(1), 29-44.

Arbuckle, J. L. (2012). AMOS 21.0. Crawforville: Amos Development Corporation.

An, Y., \& Park, S. (2017). A study on the effects of community spirit on interpersonal relationships of adolescents and the mediating effects of self-esteem. Journal of School Social Work, 39, 95-117. https://doi. org/10.20993/jSSW.39.5

Bailey, B., \& Davidson, J. (2007). Psychological and physical benefits of participation in vocal performance. In J. Edwards (Ed.), Music: Promoting health and creating community in healthcare contexts ( $p$ p. 52-63). Newcastle: Cambridge Scholars Publishing.

Bohnert, A. M., Kane, P., \& Gaber, J. (2008). Organized activity participation and internalizing and externalizing symptoms: Reciprocal relations during adolescence. Journal of Youth Adolescence, 37(2), 239-250. http://dx.doi.org/10.1007/s10964-0079195-1

Bronfenbrenner, U. (1989). Ecological systems theory. In R. Vasta (Ed.), Annals of child development, Vol. 6. Six theories of child development: Revised reformulations and current issues (pp. 187249). London: JAI Press.

Brown, T. (2007). Predicting young adult outcomes from adolescent activities and family structure: A social capital approach (Unpublished doctoral dissertation). Arizona State University, Arizona, U.S.

Chipuer, H. M. (2001). Dyadic attachments and community connectedness: Links with youths'loneliness. Journal of Community Psychology, 29(4), 429-446. https://doi.org/10.1002/jcop.1027

Cho, J. H., Lee, S. H., \&t Ko, D. H. (2014). The differences of adolescence's leisure activities depending on the parent's socio-economic status. Journal of Tourism Science, 38(5), 139-158.

Cho, M. (2007). The effects which the group spirit could have on the school life and academic achievement (Unpublished master's thesis). Chungnam National University, Daejon, Korea.

Choi, B. O. (2014). The effects of community-based creative experience activities on career-related and emotion-related factors. Teacher Education Research, 53(4), 693-705.

Choi, K. I. (2018). An effect of satisfaction at youth's experiential activities on multicultural acceptance: An mediating effect of the interpersonal relationship and sense of community. Journal of Youth Welfare, 20(1), 117-135. http://dx.doi.org/10.19034/ KAYW.2018.20.1.05

Choi, M. (2002). The relationship between volunteering service level and self-esteem of the youth (Unpublished master's thesis). Kyonggi University, Suwon, Korea.

Conrad, D., \& Hedin, D. (1989). High school community service: A review of research and programs. Wisconsin: Madison Center for Education 
Research, University of Wisconsin-Madison.

Coplan, R. J., Rose-Krasnor, L., Weeks, M., Kingsbury, A., Kingsbury, M., \& Bullock, A. (2013). Alone is a crowd: Social motivations, social withdrawal, and socioemotional functioning in later childhood. Developmental Psychology, 49(5), 861-875. https://doi.org/10.1037/ a0028861

Digital News Team. (2014, November 14). Korean children 'satisfaction of life' is lowest in OECD ... Increased stress and depression. Kyunghyang Shinmun. Retrieved October 1, 2018, from http://news.khan.co.kr/kh_ news/khan_art_view.html?code $=940702$ \&tartid $=201411041508151$

Do, J. S., \& Sung, J. M. (2013). The effects of youth activity on sociality development. Journal of Adolescent Welfare, 15(2), 145-173.

Du, H., Bernardo, A. B., \& Yeung, S. S. (2015). Locus-of-hope and life satisfaction: The mediating roles of personal self-esteem and relational self-esteem. Personality and Individual Differences, 83, 228-233. https://doi.org/10.1016/j.paid.2015.04.026

Fauth, R. C., Roth, J. L., \& Brooks-Gunn, J. (2007). Does the neighborhood context alter the link between youth's after-school time activities and developmental outcomes? A multilevel analysis, Developmental Psychology, 43(3), 760-777. https://doi.org/10.1037/00121649.43.3.760

Hall, G. S. (1904). Adolescence: Its psychology and its relations to physiology, anthropology, sociology, sex, crime, religion, and education. NY: Appleton.

Gunnar, M. R., \& Quevedo, K. (2007). The neurobiology of stress and development. Annual Review of Psychology, 58, 145-173. https://doi. org/10.1146/annurev.psych.58.110405.085605

Harrison, P. A., \& Narayan, G. (2003). Differences in behaviour, psychological; factors, and environmental factors associated with participation in school sports and other activities in adolescence. Journal of School Health, 73(3), 113-120. https://doi.org/10.1111/ j.1746-1561.2003.tb03585.x

Hong, B., \& Nam, M. (2004). Social welfare with youth. Seoul: Yangseowon.

Hong, J. (2001). The effects of voluntary service activities of middle school students on self-esteem and achievement motivation (Unpublished master's thesis). Dongguk University, Seoul, Korea.

Hong, N. (2015). The effects of youth volunteering on school adjustment mediated by self-esteem and community spirit. Forum for Youth Culture, 44, 157-180. http://dx.doi.org/10.17854/ffyc.2015.10.44.157

Hong, S. H. (2000). The criteria for selecting appropriate fit indices in structural equation modeling and their rationales. Korean Journal of Clinical Psychology, 19(1), 161-177.

Hwang, J., Huh, H., \&t An, H. (2013). Changes in youths'participation in youth activities, their self-recognition and social development: $A$ longitudinal analysis from middle school year 1 to year 3 (National
Youth Policy Institute (Report No. 13-R14-3). Seoul: National Youth Policy Institute.

Ji, S. (2006). The study of high school student's activities of Marine Sports on the development of their sociality. Journal of Fisheries and Marine Sciences Education, 18(3), 329-340.

Jung, S. B., Kim, J. T., \& Kim, E. S. (2016). Structural relationship mode among physical competence, self-esteem, sense of community and school adjustment of adolescent. Journal of the Korean Society for Wellness, 11(3), 327-343. https://doi.org/10.21097/ ksw.2016.08.11.3.327

Jwa, H., \& Oh, S. (2011). Gender differences in developmental trajectory and predictors of internalizing problem. Journal of Adolescent Welfare, 13(4), 119-147.

Kim, D. J. (2009). Influence of volunteer service upon sociality development of adolescence. Journal of Social Welfare Support, 4(1), 123-146.

Kim, G. S. (2010). Analysis of AMOS 18.0 structural equation model. Seoul: Hannarea Academy.

Kim, H. (1999). The influence of youth volunteer activity on the social development (Unpublished master's thesis). Dong-A university, Busan, Korea.

Kim, H. (2014). The effects of latent classes in adolescents' social activity participation on their sense of community. Journal of School Social Work, 28, 195-214.

Kim, J. H., \& Yoo, H. C. (2009). The same age relationship of the young people swim classroom participant affects in community spirit and social maturity degree. Journal of Sport and Leisure Studies, 35, 609619.

Kim, K. (2007). The influences of high school students' volunteer activities on their self-esteem (Unpublished doctoral dissertation). Daegu University, Daegu, Korea.

Kim, S., \& Kim, K. (1998). Development of behavior problem scale for children and adolescence. Journal of Korean Home Management Association, 16(4), 155-166.

Kim, S. S., \&t Ahn, J. J. (2012). The effects of adolescents' volunteer activities on their sense of community: Focusing on the mediating role of self-esteem and peer attachment. Korean Journal of Social Welfare Studies, 43(1), 339-363.

Kuhlberg, J. A., Pena, J. B., \& Zayas, L. H. (2010). Familism, parentadolescent conflict, self-esteem, internalizing behaviors and suicide attempts among adolescent Latinas. Child Psychiatry Human Development, 41(4), 425-440. https://doi.org/10.1007/s10578-0100179-0

Kwon, H. (2004). Study on the democratic citizenship of middle school students (Unpublished master's thesis). Ewha Womans University, Seoul, Korea. 
Family and Environment Research

Kwon, M. (2014). Effects of social withdrawal and depression on physical education record of adolescents. The Korea Journal of Sports Science, 22(3), 351-360.

Lee, J. H., Jung, J. S., \& Lee, S. J. (2014). A research on the effects of art media on adolescents'depression, anxiety, and interpersonal relationship. Journal of Arts Psychotherapy, 10(2), 263-286.

Lee, B., Min, W., \&t Kim, J. (2014). A study for developmental trajectories of social withdrawal in adolescence: An exploratory approach based on developmental-contextualism perspective. Korean Journal of Youth Studies, 21(8), 317-346.

Lee, T., Lee, S., \&t Han, Y. (2015). The moderating effect of gender in the relationship between physical education and adolescents'internalizing and externalizing problem behaviors: Using multi-level growth modeling. The Korean Journal of School Psychology: Culture and Social Issue, 21(2), 131-158.

Lindblad, F., Hogmark, A., \& Theorell, T. (2007). Music intervention for 5th and 6th graders e effects on development and cortisol secretion. Stress and Health, 23(1), 9-14. https://doi.org/10.1002/smi.1109

Mash, E. J., \&t Barkley, R. A. (2003). Child psychopathology (2nd ed). New York: Guilford.

Mcdonald, E. J., McCabe, K., Yeh, M., Lau, A., Garland, A., \& Hough, R. L. (2005). Cultural affiliation and self-esteem as predictors of internalizing symptoms among Mexican American adolescents. Journal of Clinical Child and Adolescent Psychology, 34(1), 163-171. https://doi.org/10.1207/s15374424jccp3401_15

McMillan, D. W., \& Chavis, D. M. (1986). Sense of community: A definition and theory. Journal of Community Psychology, 14(1), 6-23.

Ministry of gender equality and family of Republic of Korea. (2012). White paper on youth (Report No. 11-1383000-000102-10). Seoul: Ministry of gender equality and family of Republic of Korea.

Moon, S. H., \&t Moon, H. Y. (2009). A comparative analysis on the realities and effects of youth volunteer work. Journal of Adolescent Welfare, 11(1), 101-120.

Moon, S. H., \&t Moon, H. Y. (2010). A study on the participation situation, satisfaction and effectiveness of youth activities. Journal of Adolescent Welfare, 12(2), 95-122.

National Youth Policy Institute. (2014). Korean Children \& Youth Panel Survey (KCYPS) the first to fifth survey sample. Seoul: National Youth Policy Institute.

National Youth Policy Institute. (2014). Korean Children \& Youth Panel Survey (KCYPS) the first to fourth survey data code book. Seoul: National Youth Policy Institute.

OECD. (2017). How does KOREA compare on child well-being? Retrieved Feb 1, 2019, from https://www.oecd.org/els/family/CWBDP_Factsheet_ KOR.pdf.

Oh, W., Rubin, K. H., Bowker, J. C., Booth-LaForce, C., Rose-Krasnor,
L., \& Laursen, B. (2008). Trajectories of social withdrawal from middle childhood to early adolescence. Journal of Abnormal Child Psychology, 36(4), 553-566.

Park, A. (2014). The effect of perceived neglect on children's depressive symptoms: focusing on mediating effects of self-esteem and social withdrawal. Journal of Adolescent Welfare, 16(4), 397-416.

Park, K. W. (2014). The effects of parents' neglect and abuse on the school adjustment in adolescents: The mediating effects of social withdrawal. Korean Journal of Child Studies, 35(1), 1-15. https://doi. org/10.5723/KJCS.2014.35.1.1

Park, G. N. (2009). The effect of youth participation on a sense of community. Korean Journal of Youth Studies, 16(10), 273-306.

Park, H., \&t Hong, S. (2014). The relationships between rejection sensitivity and victimization of children: mediating effects of selfesteem, social withdrawal and inhibition. The Journal of Korea Elementary Education, 25(3), 1-18. https://doi.org/10.20972/ kjee.25.3.201409.1

Park, J., \& Lee, T. (2015). The effects of participation duration and satisfaction in youth's experiential activities on life satisfaction and sense of community: The mediating effect on school adjustment Journal of the Korea Institute of Youth Facility and Environment, 13(2), 237-248.

Park, K. W. (2014). The effects of parents' neglect and abuse on the school adjustment in adolescents: The mediating effects of social withdrawal. Korean Journal of Child Studies, 35(1), 1-15. https://doi. org/10.5723/KJCS.2014.35.1.1

Rosenberg, M. (1965). Society and the adolescent self-image. Princeton, $\mathrm{NJ}$ : Princeton University Press.

Rosenberg, M. (1979). Conceiving the self. New York: Basic Books.

Rubin, K. H., \&t Burgess, K. B. (2001). Social withdrawal and anxiety. In M. W. Vasey \&t M. R. Dadds (Eds.), The developmental psychopathology of anxiety (pp. 407-434). New York: Oxford University Press.

Rubin, K. H., Chen, X., \&t Hymel, S. (1993). Socioemotional characteristics of withdrawn and aggressive children. Merrill-Palmer Quarterly, 39(4), 518-534.

Rubin, K. H., Coplan, R. J., \& Bowker, H. C. (2009). Social withdrawal in childhood. Annual Review of Psychology, 60, 141-171. https://doi. org/10.1146/annurev.psych.60.110707.163642

Ryu, M. G. (2013). Mediating effects of self-regulation on the relationship between sociality development and participation satisfaction to the youth culture zone. Journal of Adolescent Behavior Science, 18, 113136.

Ryu, S., \&t Kang, B. (2012). The effects of adolescents'leisure experiences on self-esteem and happiness. Studies on Korean Youth, 23(4), 27-50.

Schwartz, C. E., Snidman, N., \&t Kagan, J. (1999). Adolescent social anxiety as an outcome of inhibited temperament in childhood. 
Journal of the American Academy of Child \&t Adolescent Psychiatry, 38(8), 1008-1015. https://doi.org/10.1097/00004583-19990800000017

Seo, M. (2008). The effects of gender and perceived academic achievement upon early adolescents'internalization of behavior problems: depression/anxiety. Studies on Korean Youth, 19(4), 115-138.

Seoul National University Hospital (2018). Prevalence and risk factors of psychiatric disorders in child and adolescent population -school based research- (Report No. HM16C1994). Sejong: Ministry of Health and Welfare.

Shin, Y. L. (2007). Social withdrawal and friendships in childhood. Korean Journal of Child Studies, 28(5), 193-207.

Shin, K., \&t You, S. (2013). Leisure type, leisure satisfaction and adolescents'psychological wellbeing. Journal of Pacific Rim Psychology, 7(2), 53-62. https://doi.org/10.1017/prp.2013.6

Smokowski, P. R., Rose, R. A., \& Bacallao, M. (2009). Influence of risk factors and cultural assets on Latino adolescents'trajectories of self-esteem and internalizing symptoms. Child Psychiatry Human
Development, 41(2), 133-155. https://doi.org/10.1007/s10578-0090157-6

Woo, Y. R., \&t Nho, C. R. (2014). Mediating effects of sense of community solidarity between emotional and aggressive problems and school adjustments among adolescents. Journal of Adolescent Welfare, 16(2), 203-227.

Seo, U. (2007). Impacts of the socioeconomic environments on the leisure activities of the youth. Studies on Korean Youth, 18(1), 273-302.

Yang, Y., \&t Kim, J. (2015). Relationship between children's self-esteem, sense of community, and multicultural receptivity. Journal of Youth Welfare, 17(4), 309-328. https://doi.org/10.19034/KAYW.2015.17.4.14

Yang, J. W., Kim, J. H., \&t Oh, K. J. (2006). Selective attentional bias for negative emotional faces in social anxiety: comparison of three age groups. Korean Journal of Clinical Psychology, 25, 237-255.

Yoon, C. (2011). Adolescent activity participations and the civic mind of adolescents: Focusing on Kwangmyeong city adolescents (Unpublished master's thesis). Chungang University, Seoul, Korea. 
